\title{
Model reduction of discrete time hybrid systems: A structural approach based on observability
}

\author{
Riccardo Vignali and Maria Prandini \\ Dipartimento di Elettronica, Informazione e Bioingegneria \\ Politecnico di Milano, Italy \\ Email: $\{$ riccardomaria.vignali, maria.prandini\}@ polimi.it
}

\begin{abstract}
This paper addresses model reduction for discrete time hybrid systems that are described by a Mixed Logical Dynamical (MLD) model. The goal is to simplify the MLD model while preserving its input/output behavior. This is useful when considering a reachability property that depends on the output and should be enforced by appropriately setting the input. The proposed procedure for model reduction rests on the analysis of the structure of the MLD system and on its observability properties. It is also applicable to PieceWise Affine (PWA) systems that can be equivalently represented as MLD systems. In the case of PWA systems, mode merging can be adopted to further simplify the model.
\end{abstract}

\section{INTRODUCTION}

In this paper we consider the problem of model reduction for discrete time hybrid systems. We focus on the class of Mixed Logical Dynamical (MLD) systems originally introduced in [4]. MLD systems are equivalent to various classes of hybrid models [2], [9], and, in particular, to PieceWise Affine (PWA) systems commuting between a finite set of affine dynamics (the modes), each one associated with a polyhedral region in the partitioned state cross input space. Various analysis and design problems have been addressed for this class of systems using an optimization-based perspective with a mixed integer programming formulation, see e.g. [3], [5], [6], [13], [15], [16]

The goal is to simplify the structure of the system while preserving its input/output behavior. This is particularly useful when addressing a reachability problem where the input has to be designed so as to satisfy some specification expressed in terms of the output evolution, or, more generally, when addressing analysis or design problems that concern the output.

To achieve our goal, we introduce a structural approach based on observability-like analysis. The notion of observability for MLD systems has been treated extensively in [2], where the concept of incrementally observable MLD system is introduced. Possible impact of observability analysis on model reduction is mentioned in the conclusions of the related paper [8]. Here, we propose an approach to model reduction that rests on the Kalman canonical decomposition into observable and unobservable part of the affine dynamics appearing in the MLD model description, which can be isolated by neglecting the discrete component of the hybrid dynamics. The soobtained seemingly unobservable components may actually affect the discrete mechanism underlying the hybrid system

This work is partly supported by the European Commission under project UnCoVerCPS with grant number 643921.

978-1-5090-3079-8/16/\$31.00 (C)2016 IEEE evolution and, hence, they may become observable. We then introduce a sufficient condition to determine if the unobservable components of the affine dynamics remains unobservable in the hybrid system dynamics. The approach applies to MLD systems and their equivalent PWA counterpart. If the obtained reduced MLD system is mapped into a PWA system (e.g., via the approach in [1]) that has the same dynamics in adjacent regions of the state cross input space, a mode aggregation procedure can be applied to further simplify the PWA model.

The proposed approach is conceptually simple and easy to implement, since it is based on the standard notion of observability for linear systems. Model reduction methods that preserve the input/output behavior of a PWA system have been proposed in the literature but in a continuous time setting, [12]. These approaches are, hence, not directly comparable with our discrete time method.

It is worth noticing that the work in this paper strictly relates to minimal realization theory in that the MLD systems is simplified while preserving exactly its input/output behavior. In the literature, minimal realization theory has been mainly developed for linear and bilinear switched and hybrid systems with externally induced switching. Apparently, it remains an open problem when considering hybrid systems with endogenous switching (see [11]). Our paper can hence be seen as a preliminary step in this direction.

The rest of the paper is structured as follows. In Section II, we describe the modeling context, recalling the equivalence between MLD and PWA systems that was proven in [2]. We then illustrate the proposed approach for model reduction based on observability-like analysis in Section III. We describe the mode reduction procedure in Section IV. We present some numerical examples in Section $\mathrm{V}$ and conclude the paper with some remarks in Section VI.

\section{MODELING FRAMEWORK}

We consider a Mixed Logical Dynamical (MLD) system described by the following inequalities:

$$
\begin{aligned}
& x(k+1)=A x(k)+B_{u} u(k)+B_{\delta} \delta(k)+B_{z} z(k)+B_{a f f} \\
& y(k)=C x(k)+D_{u} u(k)+D_{\delta} \delta(k)+D_{z} z(k)+D_{a f f} \\
& E_{x} x(k)+E_{u} u(k)+E_{\delta} \delta(k)+E_{z} z(k) \leq E_{a f f}
\end{aligned}
$$

where $x \in \mathbb{R}^{n_{c}} \times\{0,1\}^{n_{l}}$ is the state composed of both continuous and binary variables, $u \in \mathbb{R}^{m_{c}} \times\left\{\begin{array}{ll}0, & 1\end{array}\right\}^{m_{l}}$ is the input vector comprising a continuous and a discrete 
component. As for $\delta$ and $z$, they are binary and continuousvalued auxiliary variables: $\delta \in\{0,1\}^{r_{l}}$ and $z \in \mathbb{R}^{r_{c}}$.

We assume that some reachability specification is given in terms of the behavior in time of the output $y \in \mathbb{R}^{p_{c}} \times\{0,1\}^{p_{l}}$.

For an MLD system to be well-defined, the solution to the inequalities in (1) must be unique, i.e., given a state-input pair there exists a unique value for the auxiliary variables $\delta$ and $z$ satisfying such inequalities.

Without loss of generality, we shall assume next that the affine terms $B_{a f f}$ and $D_{a f f}$ are both zero. Indeed, if this were not the case, one can introduce $\bar{x}(k)$ and $\bar{y}(k)$ given by the solution to the system

$$
\begin{aligned}
& \bar{x}(k+1)=A \bar{x}(k)+B_{a f f} \\
& \bar{y}(k)=C \bar{x}(k)+D_{a f f}
\end{aligned}
$$

and replace $x$ and $y$ in (1) with $x+\bar{x}$ and $y+\bar{y}$. As a result, the affine terms will cancel out and the right hand side of the last inequality in (1) will become $E_{a f f}-E_{x} \bar{x}(k)$. If $I-A$ is invertible and one can choose $\bar{x}(0)=(I-A)^{-1} B_{a f f}$, then, the solution $\bar{x}(k)$ and $\bar{y}(k)$ to (2) keep constant and, hence, $E_{a f f}$ in (1) is replaced by a time invariant term $E_{a f f}-E_{x} \bar{x}(k)=$ $E_{a f f}-E_{x}(I-A)^{-1} B_{a f f}$.

Let us consider a PieceWise Affine (PWA) systems governed by

$$
\begin{aligned}
x(k+1) & =A_{i} x(k)+B_{i} u(k)+f_{i} \\
y(k) & =C_{i} x(k)+D_{i} u(k)+g_{i}
\end{aligned} \quad \text { for }\left[\begin{array}{c}
x(k) \\
u(k)
\end{array}\right] \in \mathcal{A}_{i},
$$

where $x \in \mathcal{X} \subseteq \mathbb{R}^{n}$ is the state, $u \in \mathcal{U} \subseteq \mathbb{R}^{m}$ is the input, and $y \in \mathbb{R}^{p}$ is the output, whereas $f_{i}, g_{i}$ are constant vectors. Suppose that the collection of sets $\{\mathcal{A}\}_{i=1}^{s}$ forms a polyhedral subdivision of the space $\mathcal{X} \times \mathcal{U}$, that is if $\cup_{i=1}^{s} \mathcal{A}_{i}=\mathcal{X} \times \mathcal{U}$, each $\mathcal{A}_{i}$ is of dimension $n+m$, and the intersection $\mathcal{A}_{i} \cap \mathcal{A}_{j}$, $i \neq j$, is either empty or a common proper face of both polyhedra. Then, system (4) is well posed and if $\mathcal{X}$ and $\mathcal{U}$ are bounded, it can be converted in an equivalent MLD system. The idea behind the conversion of a PWA system into the MLD form is to introduce auxiliary variables $\delta$ and $z$ that respectively capture which of the original PWA mode is active, and the dynamics associated to that mode. This is done by means of big- $M$ techniques that lead to the linear inequalities in (1) (see [2]). The MLD form is typically more convenient when performing optimization-based analysis and design. Notably, the opposite implication also holds true, i.e., MLD systems have an equivalent PWA form.

\section{STRUCTURAL REDUCTION}

Our aim is to detect whether there exists some part of the MLD system (1) that can be neglected without affecting the output behavior.

We start by considering the simple case when the MLD system (1) reduces to a standard linear system, i.e.,

$$
\begin{aligned}
& x(k+1)=A x(k)+B_{u} u(k) \\
& y(k)=C x(k)+D_{u} u(k) .
\end{aligned}
$$

In this setting, we just need to determine the non-observable part of the system and then remove it, which entails neglecting those inputs that do not affect the observable part (noninfluential inputs). This is achieved via a three-steps procedure:

1) Rewrite the system in its observable canonical form by means of an appropriate similarity transformation $T_{o}$ :

$$
\begin{aligned}
& {\left[\begin{array}{c}
x_{n o}(k+1) \\
x_{o}(k+1)
\end{array}\right]=\tilde{A}\left[\begin{array}{c}
x_{n o}(k) \\
x_{o}(k)
\end{array}\right]+\tilde{B}_{u} u(k),} \\
& y(k)=\tilde{C}\left[\begin{array}{c}
x_{n o}(k) \\
x_{o}(k)
\end{array}\right]+D_{u} u(k),
\end{aligned}
$$

where $\left[\begin{array}{c}x_{n o} \\ x_{o}\end{array}\right]=T_{o} x, \quad x_{n o} \in \mathbb{R}^{\nu_{n o}}, x_{o} \in \mathbb{R}^{\nu_{o}}, \tilde{B}=$ $T_{o} B_{u}, \tilde{C}=C T_{o}^{-1}=\left[\begin{array}{ll}0 & C_{o}\end{array}\right]$ and $\tilde{A}$ has the following upper triangular structure:

$$
\tilde{A}=\left[\begin{array}{cc}
A_{n o} & A_{12} \\
0 & A_{o}
\end{array}\right] .
$$

2) Remove the non-observable state component $x_{n o}$, i.e., remove the first $\nu_{n o}$ rows of $\tilde{A}, \tilde{B}_{u}$ and the first $\nu_{n o}$ columns of $\tilde{C}$. The resulting system is given by:

$$
\begin{aligned}
& x_{o}(k+1)=A_{o} x_{o}(k)+\tilde{B}_{u, o} u(k), \\
& y(k)=C_{o} x_{o}(k)+D_{u} u(k),
\end{aligned}
$$

where $\tilde{B}_{u, o}$ is the matrix obtained by extracting the last $\nu_{o}$ rows of $\tilde{B}_{u}$.

3) Check if there exists an index $j \in\{1, \ldots, m\}$ such that the $j$-th column of both $\tilde{B}_{u, o}$ and $D_{u}$ are null; if that is the case, input $u_{j}$ is non-influential and can be removed.

Note that, by construction, the evolution of the output of the reduced order system (6) coincides with the evolution of the output of the original system (5), for any initial condition, and for any assignment of the input.

Our aim now is to detect and remove the non-observable part of the system in the case when some discrete dynamics is present. For sake of simplicity, we consider MLD systems without logic states, i.e., $x \in \mathbb{R}^{n_{c}}$. We rewrite the MLD system (1) with $B_{a f f}=0$ and $D_{a f f}=0$ and the time index dropped for convenience:

$$
\begin{aligned}
& x^{+}=A x+B_{u} u+B_{\delta} \delta+B_{z} z \\
& y=C x+D_{u} u+D_{\delta} \delta+D_{z} z \\
& E_{x} x+E_{u} u+E_{\delta} \delta+E_{z} z \leq E_{a f f} .
\end{aligned}
$$

We start focusing on matrices $A, B_{u}, C, D_{u}$, as if the system were linear, and compute the similarity transformation $T_{o}$ as in the linear case. The system become:

$$
\begin{aligned}
& {\left[\begin{array}{c}
x_{n o} \\
x_{o}
\end{array}\right]^{+}=\tilde{A}\left[\begin{array}{c}
x_{n o} \\
x_{o}
\end{array}\right]+\tilde{B}_{u} u+\tilde{B}_{\delta} \delta+\tilde{B}_{z} z} \\
& y=\tilde{C}\left[\begin{array}{c}
x_{n o} \\
x_{o}
\end{array}\right]+D_{u} u+D_{\delta} \delta+D_{z} z \\
& \tilde{E}_{x}\left[\begin{array}{c}
x_{n o} \\
x_{o}
\end{array}\right]+E_{u} u+E_{\delta} \delta+E_{z} z \leq E_{a f f}
\end{aligned}
$$


where $\tilde{A}$ and $\tilde{C}$ are defined as in the previous section and $\tilde{B}_{u}=T_{o} B_{u}, \tilde{B}_{\delta}=T_{o} B_{\delta}, \tilde{B}_{z}=T_{o} B_{z}, \tilde{E}_{x}=E_{x} T_{o}^{-1}$.

Despite the structure of matrices $\tilde{A}$ and $\tilde{C}$, before possibly removing $x_{n o}$, we need first to check if $x_{n o}$ affects the output via the inequalities (10). To understand why this might be the case, suppose that $x_{n o}$ affects the value of $\delta$ via the inequalities (10), then, $x_{n o}$ is indirectly influencing the output via the term $D_{\delta} \delta$. However, it may be also the case that $x_{n o}$ affects only those elements of $\delta$ that are "hidden" by matrix $D_{\delta}$, so that in the end $x_{n o}$ does not affect the output. For this reason we should check if $x_{n o}$ affects $\tilde{\tilde{\delta}}=D_{\delta} \delta$ instead of $\delta$. This consideration applies also to variables $u$ and $z$, so that before analyzing the dependencies introduced by the inequalities (10) we need first to set some changes of variables.

To make the discussion as general as possible we consider the general case when $D_{u}, D_{\delta}$ and $D_{z}$ may be rank deficient matrices, i.e.: $\operatorname{rank}\left(D_{u}\right)=\mathbf{r}_{u} \leq \min \{p, m\}, \operatorname{rank}\left(D_{\delta}\right)=$ $\mathbf{r}_{\delta} \leq \min \left\{p, r_{l}\right\}, \operatorname{rank}\left(D_{z}\right)=\mathbf{r}_{z} \leq \min \left\{p, r_{c}\right\}$.

The full rank factorization (see [7]) of $D_{u}, D_{\delta}$ and $D_{z}$ : $D_{u}=D_{u, L} D_{u, R}, D_{\delta}=D_{\delta, L} D_{\delta, R}, D_{z}=D_{z, L} D_{z, R}$, where $D_{u, L}, D_{\delta, L}, D_{z, L}$ have, respectively, $\mathbf{r}_{u}, \mathbf{r}_{\delta}, \mathbf{r}_{z}$ columns, can be used to introduce the following change of variables:

$$
\left[\begin{array}{c}
\tilde{u} \\
\tilde{u}^{\perp}
\end{array}\right]=\left[\begin{array}{c}
D_{u, R} \\
F_{u}
\end{array}\right] u, \quad\left[\begin{array}{c}
\tilde{\delta} \\
\tilde{\delta}^{\perp}
\end{array}\right]=\left[\begin{array}{c}
D_{\delta, R} \\
F_{\delta}
\end{array}\right] \delta, \quad\left[\begin{array}{c}
\tilde{z} \\
\tilde{z}^{\perp}
\end{array}\right]=\left[\begin{array}{c}
D_{z, R} \\
F_{z}
\end{array}\right] z,
$$

where each row of matrix $F_{i}, i \in\{u, \delta, z\}$, is orthogonal to each row of the corresponding matrix $D_{i, R}$ (i.e., the rows of $F_{i}$ form a basis of the null space of $D_{i, R}$ ). Note that the resulting matrices $P_{i}$ defined as $P_{i}=\left[D_{i, R}^{\prime} F_{i}^{\prime}\right]^{\prime}, i \in\{u, \delta, z\}$ are square and invertible by construction.

In view of the change of variables in (11), the system can be rewritten as:

$$
\begin{aligned}
& {\left[\begin{array}{c}
x_{n o} \\
x_{o}
\end{array}\right]^{+}=\tilde{A}\left[\begin{array}{c}
x_{n o} \\
x_{o}
\end{array}\right]+\left[\begin{array}{ll}
B_{u, L}^{*} & B_{u, R}^{*}
\end{array}\right]\left[\begin{array}{c}
\tilde{u} \\
\tilde{u}^{\perp}
\end{array}\right]} \\
& +\left[\begin{array}{ll}
B_{\delta, L}^{*} & B_{\delta, R}^{*}
\end{array}\right]\left[\begin{array}{c}
\tilde{\delta} \\
\tilde{\delta}^{\perp}
\end{array}\right]+\left[\begin{array}{ll}
B_{z, L}^{*} & B_{z, R}^{*}
\end{array}\right]\left[\begin{array}{c}
\tilde{z} \\
\tilde{z}^{\perp}
\end{array}\right] \\
& y=\tilde{C}\left[\begin{array}{c}
x_{n o} \\
x_{o}
\end{array}\right]+D_{u, L} \tilde{u}+D_{\delta, L} \tilde{\delta}+D_{z, L} \tilde{z} \\
& \tilde{E}_{x}\left[\begin{array}{c}
x_{n o} \\
x_{o}
\end{array}\right]+\left[E_{u, L}^{*} E_{u, R}^{*}\right]\left[\begin{array}{c}
\tilde{u} \\
\tilde{u}^{\perp}
\end{array}\right]+\left[\begin{array}{ll}
E_{\delta, L}^{*} & E_{\delta, R}^{*}
\end{array}\right]\left[\begin{array}{c}
\tilde{\delta} \\
\tilde{\delta}^{\perp}
\end{array}\right] \\
& +\left[\begin{array}{c}
E_{z, L}^{*} E_{z, R}^{*}
\end{array}\right]\left[\begin{array}{c}
\tilde{z} \\
\tilde{z}^{\perp}
\end{array}\right] \leq E_{a f f},
\end{aligned}
$$

where we set

$$
\begin{array}{rlrl}
{\left[\begin{array}{ll}
B_{u, L}^{*} & B_{u, R}^{*}
\end{array}\right]} & =P_{u}^{-1} \tilde{B}_{u} & & {\left[\begin{array}{ll}
E_{u, L}^{*} & E_{u, R}^{*}
\end{array}\right]=P_{u}^{-1} E_{u}} \\
{\left[\begin{array}{ll}
B_{\delta, L}^{*} & B_{\delta, R}^{*}
\end{array}\right]=P_{\delta}^{-1} \tilde{B}_{\delta}} & {\left[\begin{array}{ll}
E_{\delta, L}^{*} & E_{\delta, R}^{*}
\end{array}\right]=P_{u}^{-1} E_{\delta}} \\
{\left[\begin{array}{ll}
B_{z, L}^{*} & B_{z, R}^{*}
\end{array}\right]=P_{z}^{-1} \tilde{B}_{z}} & {\left[\begin{array}{ll}
E_{z, L}^{*} & E_{z, R}^{*}
\end{array}\right]=P_{z}^{-1} E_{z} .}
\end{array}
$$

Finally, by defining variables $\tilde{\tilde{u}}, \tilde{\tilde{\delta}}, \tilde{\tilde{z}}$ as:

$$
\tilde{\tilde{u}}=D_{u, L} \tilde{u}, \quad \tilde{\tilde{\delta}}=D_{\delta, L} \tilde{\delta}, \quad \tilde{\tilde{z}}=D_{z, L} \tilde{z},
$$

the system can be rewritten as:

$$
\begin{aligned}
& {\left[\begin{array}{c}
x_{n o} \\
x_{o}
\end{array}\right]^{+}=\tilde{A}\left[\begin{array}{c}
x_{n o} \\
x_{o}
\end{array}\right]+\left[\begin{array}{ll}
\bar{B}_{u} & B_{u, R}^{*}
\end{array}\right]\left[\begin{array}{c}
\tilde{\tilde{u}} \\
\tilde{u}^{\perp}
\end{array}\right]} \\
& +\left[\begin{array}{ll}
\bar{B}_{\delta} & B_{\delta, R}^{*}
\end{array}\right]\left[\begin{array}{c}
\tilde{\tilde{\delta}} \\
\tilde{\delta}^{\perp}
\end{array}\right]+\left[\begin{array}{ll}
\bar{B}_{z} & B_{z, R}^{*}
\end{array}\right]\left[\begin{array}{c}
\tilde{\tilde{z}} \\
\tilde{z}^{\perp}
\end{array}\right] \\
& y=\tilde{C}\left[\begin{array}{c}
x_{n o} \\
x_{o}
\end{array}\right]+\tilde{\tilde{u}}+\tilde{\tilde{\delta}}+\tilde{\tilde{z}} \\
& \tilde{E}_{x}\left[\begin{array}{c}
x_{n o} \\
x_{o}
\end{array}\right]+\left[\begin{array}{ll}
\bar{E}_{u} & E_{u, R}^{*}
\end{array}\right]\left[\begin{array}{c}
\tilde{\tilde{u}} \\
\tilde{u}^{\perp}
\end{array}\right]+\left[\begin{array}{ll}
\bar{E}_{\delta} & E_{\delta, R}^{*}
\end{array}\right]\left[\begin{array}{c}
\tilde{\tilde{\delta}} \\
\tilde{\delta}^{\perp}
\end{array}\right] \\
& +\left[\begin{array}{ll}
\bar{E}_{z} & E_{z, R}^{*}
\end{array}\right]\left[\begin{array}{c}
\tilde{\tilde{z}} \\
\tilde{z}^{\perp}
\end{array}\right] \leq E_{a f f}
\end{aligned}
$$

where

$$
\begin{aligned}
\bar{B}_{u} & =B_{u, L}^{*} D_{u, L}^{\dagger} & \bar{E}_{u} & =E_{u, L}^{*} D_{u, L}^{\dagger} \\
\bar{B}_{\delta} & =B_{\delta, L}^{*} D_{\delta, L}^{\dagger} & \bar{E}_{\delta} & =E_{\delta, L}^{*} D_{\delta, L}^{\dagger} \\
\bar{B}_{z} & =B_{z, L}^{*} D_{z, L}^{\dagger} & \bar{E}_{z} & =E_{z, L}^{*} D_{z, L}^{\dagger}
\end{aligned}
$$

and $Q^{\dagger}$ denotes the left Moore-Penrose pseudoinverse of $Q$, i.e., $Q^{\dagger}=\left(Q^{\prime} Q\right)^{-1} Q^{\prime}$. Note that the transformations (11) and (12) can be combined, thus leading to:

$$
\left[\begin{array}{c}
\tilde{\tilde{u}} \\
\tilde{u}^{\perp}
\end{array}\right]=T_{u} u, \quad\left[\begin{array}{c}
\tilde{\delta} \\
\tilde{\delta}^{\perp}
\end{array}\right]=T_{\delta} \delta, \quad\left[\begin{array}{c}
\tilde{z} \\
\tilde{z}^{\perp}
\end{array}\right]=T_{z} z,
$$

where matrices $T_{u}, T_{\delta}$ and $T_{z}$ are given by

$$
T_{u}=\left[\begin{array}{c}
D_{u} \\
F_{u}
\end{array}\right], \quad T_{\delta}=\left[\begin{array}{c}
D_{\delta} \\
F_{\delta}
\end{array}\right], \quad T_{z}=\left[\begin{array}{c}
D_{z} \\
F_{z}
\end{array}\right]
$$

and have all full column rank by construction. Note that the above transformation highlights $\tilde{\tilde{u}}, \tilde{\tilde{\delta}}$, $\tilde{\tilde{z}}$, which represent the linear combinations of elements of the original vectors $u, \delta$, $z$ that affect the output of system (7).

Based on (13), (14), (15), we can now carry out the removal of those parts of the system that do not affect the output. To this purpose, we propose the following procedure.

1) Construct the undirected graph $\mathcal{G}$ of dependencies among the components of $x_{n o}, x_{o}, \tilde{\tilde{u}}, \tilde{u}^{\perp}, \tilde{\tilde{\delta}}, \tilde{\delta} \tilde{\delta}^{\perp}, \tilde{\tilde{z}}, \tilde{z}^{\perp}$ induced by inequalities (15). In particular, define as the nodes of $\mathcal{G}$ such components and draw an arc between two nodes if there is a scalar inequality in (15) involving the corresponding variables.

2) Build vector $\hat{x}_{n o}$ with the components of $x_{n o}$ that are not connected via a path of $\mathcal{G}$ to any component of $x_{o}$, $\tilde{\tilde{u}}, \tilde{\tilde{\delta}}, \tilde{\tilde{z}}$.

3) Collect in $\hat{\tilde{u}}^{\perp}$ the components of $\tilde{u}^{\perp}$, whose corresponding column in $B_{u, R}^{*}$ is null and that are not connected via a path of $\mathcal{G}$ to any component of $x_{o}, \tilde{\tilde{u}}, \tilde{\tilde{\delta}}, \tilde{\tilde{z}}$. Similarly, define $\hat{\tilde{\delta}}^{\perp}$ and $\hat{\tilde{z}}^{\perp}$.

4) Remove from (13) all state equations corresponding to the elements of $\hat{x}_{n o}$. Accordingly, remove also the corresponding columns of $\tilde{A}, \tilde{C}, \tilde{E}_{x}$. 
5) Remove from $\tilde{u}^{\perp}$ the components in $\hat{\tilde{u}}^{\perp}$ and remove the corresponding columns in $B_{u, R}^{*}$ and $E_{u, R}^{*}$. Proceed in the same way for the components of $\hat{\tilde{\delta}}^{\perp}$ and $\hat{\tilde{z}}^{\perp}$.

6) Remove from the transformation matrix $T_{u}$ the rows corresponding to the components in $\hat{\tilde{u}}^{\perp}$. If the resulting matrix has a column $j$ which is identically 0 , then the associated original input $u_{j}$ is non-influential, and, hence, can be neglected.

Note that the procedure described above can be carried out with very little computational effort, since it only requires the computation of the paths on a graph, which is an operation for which extremely efficient methods exist. Also, it is not affected by $E_{a f f}$, so that the fact that $E_{a f f}$ may be time varying is not an issue.

\section{REMOVAL OF REDUNDANT MODES}

As mentioned in Section II, if an MLD system is well-posed, then, it can be converted in an equivalent PWA system.

It may be the case that, after the model reduction performed on the MLD system, some modes in the PWA form share the same dynamics. In these cases it may be convenient to merge them, so as to reduce the total number of modes in the PWA model. The PWA representation (4) requires the sets $\mathcal{A}_{i}$ to form a polyhedral subdivision of the state-input space. For this reason, in the proposed mode merging approach, we first detect the subsets of modes that share the same dynamics, then we check if there exists a pair of modes such that their union is convex and, if so, we merge them. The resulting set becomes a new element of the subset of modes that share that same dynamics, and the exploration continues iteratively. The test of the convexity of the union of two sets, and their actual merging can be performed via the Multi Parametric Toolbox (MPT) [10]. Note that the order followed in the merging of the modes matters, as it is shown in Figure 1. One can opt for a greedy exploration which is sub-optimal in terms of number of modes merged but it is less time consuming, or an exhaustive exploration, which merges the maximum number of modes but it is more time consuming.

\section{NUMERICAL EXAMPLES}

In this section we present two examples of model reduction: Section V-A refers to the procedure for structural reduction in Section III, and Section V-B illustrates some results of the modes merging algorithm in Section IV.

\section{A. Structural reduction}

We next show the effectiveness of the approach described in Section III via a numerical example. Consider the MLD system described by:

$$
\begin{aligned}
& x(k+1)=A x(k)+B_{u} u(k)+B_{z} z(k) \\
& y(k)=C x(k)+D_{u} u(k)+D_{z} z(k) \\
& E_{x} x(k)+E_{u} u(k)+E_{\delta} \delta(k)+E_{z} z(k) \leq E_{a f f}
\end{aligned}
$$
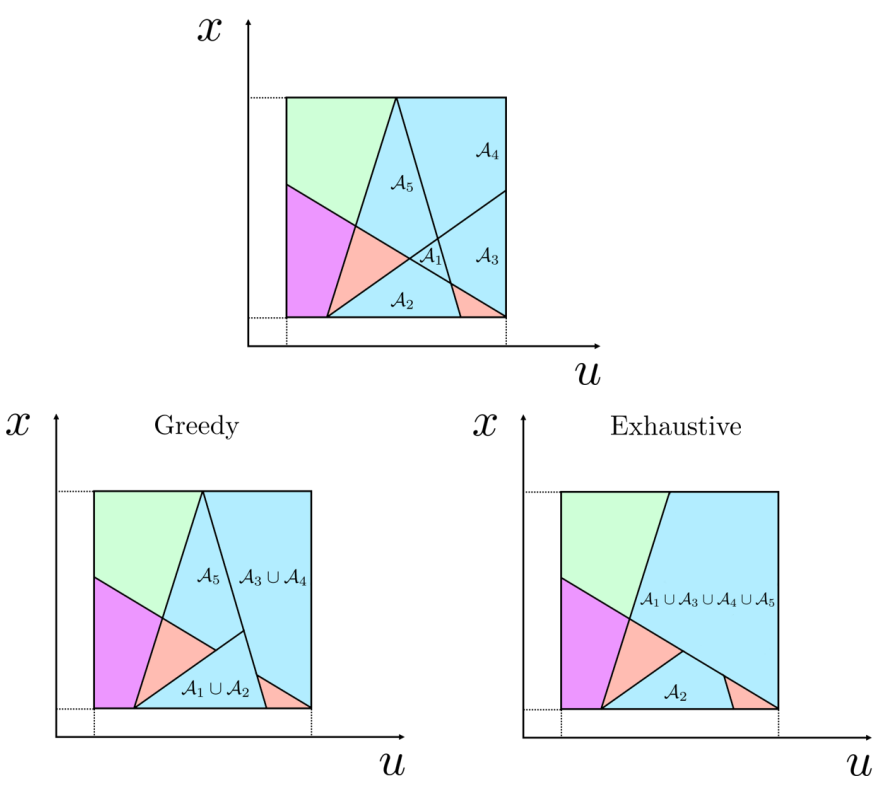

Figure 1. Pictorial view of the difference between a greedy merging routine and an exhaustive one. In the greedy routine we merge a mode with the first mode found that makes the union convex. Merging $\mathcal{A}_{1}$ with $\mathcal{A}_{2}$ generates a region that can not be merged neither with $\mathcal{A}_{5}$ nor with the union of $\mathcal{A}_{3}$ and $\mathcal{A}_{4}$. Thus, the total number of obtained regions that share the same dynamics is 3 . On the other hand, an exhaustive exploration is able to construct only two regions.

where

$$
\begin{aligned}
& A=\left[\begin{array}{cccc}
1 & 0 & -2 & 0 \\
0 & 1 & 0 & 0 \\
-3 & 0 & -4 & 0 \\
0 & 0 & 0 & 1
\end{array}\right], \quad C=\left[\begin{array}{llll}
1 & 0 & 0 & 0 \\
0 & 1 & 1 & 0
\end{array}\right], \\
& B_{u}=\left[\begin{array}{lllll}
1 & 0 & 0 & 0 & 0 \\
1 & 0 & 2 & 0 & 0 \\
1 & 3 & 0 & 0 & 0 \\
0 & 0 & 0 & 1 & 1
\end{array}\right], \quad B_{z}=\left[\begin{array}{llll}
1 & 0 & 0 & 0 \\
0 & 1 & 0 & 0 \\
0 & 0 & 1 & 0 \\
0 & 0 & 0 & 1
\end{array}\right], \\
& D_{u}=\left[\begin{array}{lllll}
1 & 0 & 0 & 0 & 0 \\
0 & 0 & 0 & 0 & 0
\end{array}\right] \quad D_{z}=\left[\begin{array}{cccc}
-1 & 0 & 1 & 0 \\
0 & 0 & 2 & 0
\end{array}\right]
\end{aligned}
$$

and the matrices of the linear inequalities in (16) are defined according to the following relations (we refer to the HYSDEL notation, see [14] )

$$
\begin{array}{ll}
\delta_{1}=u_{1} \leq 5 & \text { if } \delta_{1} \text { then } x_{1} \text { else } x_{1}+x_{3} \\
\delta_{2}=u_{2} \leq 5 & \text { if } \delta_{2} \text { then } 2 x_{2} \text { else }-x_{1}-x_{2} \\
\delta_{3}=u_{3} \leq 5 & \text { if } \delta_{3} \text { then } x_{1}-x_{3} \text { else }-x_{3} \\
\delta_{4}=u_{4} \leq 5 & \text { if } \delta_{4} \text { then }-x_{4} \text { else } 2 x_{4}
\end{array}
$$

We aim at obtaining a reduced order system, that preserves the input/output behavior of (16). To this end, we apply the procedure described in Section III and obtain:

1 State variables eliminated: $x_{4}$

2 Non-influential input variables found:

$u_{4}, u_{5}$

2 Auxiliary variables eliminated: $\delta_{4}, z_{4}$ 
so that the resulting system is described by:

$$
\begin{aligned}
& A_{\text {red }}=\left[\begin{array}{ccc}
1 & 0 & -2 \\
0 & 1 & 0 \\
-3 & 0 & -4
\end{array}\right], C_{\text {red }}=\left[\begin{array}{lll}
1 & 0 & 0 \\
0 & 1 & 1
\end{array}\right], \\
& B_{u, \text { red }}=\left[\begin{array}{lll}
1 & 0 & 0 \\
1 & 0 & 2 \\
1 & 3 & 0
\end{array}\right], \quad B_{z, \text { red }}=\left[\begin{array}{lll}
1 & 0 & 0 \\
0 & 1 & 0 \\
0 & 0 & 1
\end{array}\right], \\
& D_{u, \text { red }}=\left[\begin{array}{lll}
1 & 0 & 0 \\
0 & 0 & 0
\end{array}\right] \quad D_{z, \text { red }}=\left[\begin{array}{ccc}
-1 & 0 & 1 \\
0 & 0 & 2
\end{array}\right]
\end{aligned}
$$

with matrices $E_{x, \text { red }}, E_{u, \text { red }}, E_{\delta \text {,red }}, E_{z, \text { red }}, E_{a f f \text {,red }}$ defined by:

$$
\begin{array}{ll}
\delta_{1}=u_{1} \leq 5 & \text { if } \quad \delta_{1} \text { then } x_{1} \text { else } x_{1}+x_{3} \\
\delta_{2}=u_{2} \leq 5 & \text { if } \quad \delta_{2} \text { then } 2 x_{2} \text { else }-x_{1}-x_{2} \\
\delta_{3}=u_{3} \leq 5 & \text { if } \quad \delta_{3} \text { then } x_{1}-x_{3} \text { else }-x_{3} .
\end{array}
$$

Note that $u_{4}$, and $u_{5}$ were found to be non-influential inputs. This means that they will not affect the output behavior and hence can be removed.

\section{B. Modes merging}

Consider the following PWA system:

$$
\left[\begin{array}{l}
x_{1}^{+} \\
x_{2}^{+}
\end{array}\right]= \begin{cases}{\left[\begin{array}{ll}
1 & 0 \\
0 & 1
\end{array}\right]\left[\begin{array}{l}
x_{1} \\
x_{2}
\end{array}\right]+\left[\begin{array}{l}
1 \\
0
\end{array}\right] u, \quad\left[\begin{array}{l}
x_{1} \\
x_{2} \\
u
\end{array}\right] \in \cup_{i=1}^{6} \mathcal{A}_{i}} \\
{\left[\begin{array}{ll}
0 & 1 \\
1 & 1
\end{array}\right]\left[\begin{array}{l}
x_{1} \\
x_{2}
\end{array}\right]+\left[\begin{array}{l}
0 \\
2
\end{array}\right] u, \quad\left[\begin{array}{c}
x_{1} \\
x_{2} \\
u
\end{array}\right] \in \mathcal{A}_{7} \cup \mathcal{A}_{8},}\end{cases}
$$

where $\left(x_{1}, x_{2}\right) \in \mathcal{X}=[-100,100]^{2}, u \in \mathcal{U}=[-10,10]$ and the sets $\mathcal{A}_{i}, i=1, \ldots, 8$ are the elements of the partition of the space $\mathcal{X} \times \mathcal{U}$ defined by the following inequalities (see Figure 2):

$$
\begin{aligned}
& x_{1} \leq 0 \\
& x_{2}-x_{1} \leq 2 \\
& u \leq 2 .
\end{aligned}
$$

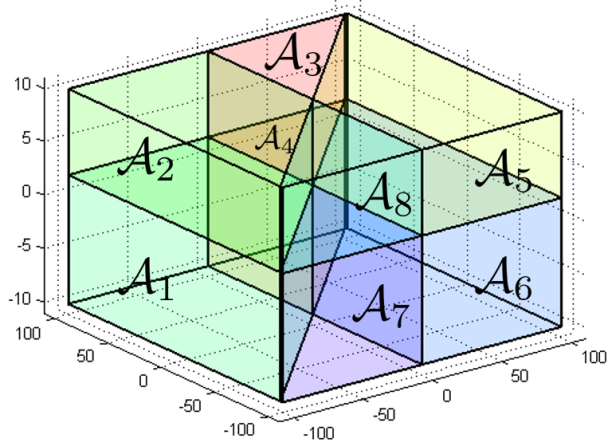

Figure 2. Partition of space $\mathcal{X} \times \mathcal{U}$ defined by inequalities (18)
We now exploit the procedure described in Section IV to merge the modes associated to the same dynamics. We group the modes in the two sets $\left\{\mathcal{A}_{1}, \mathcal{A}_{2}, \mathcal{A}_{3}, \mathcal{A}_{4}, \mathcal{A}_{5}, \mathcal{A}_{6}\right\}$ and $\left\{\mathcal{A}_{7}, \mathcal{A}_{8}\right\}$ and perform the merging on each of them. The results are depicted in Figure 2 and Figure 4.
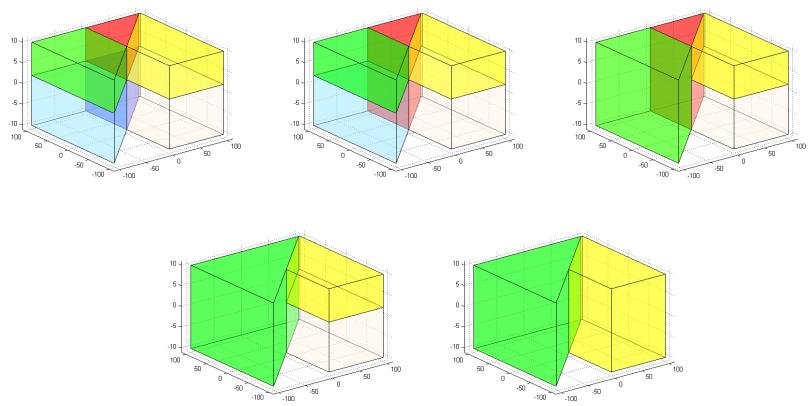

Figure 3. Merging of modes $\mathcal{A}_{1}, \mathcal{A}_{2}, \mathcal{A}_{3}, \mathcal{A}_{4}, \mathcal{A}_{5}, \mathcal{A}_{6}$ associated to the first dynamics
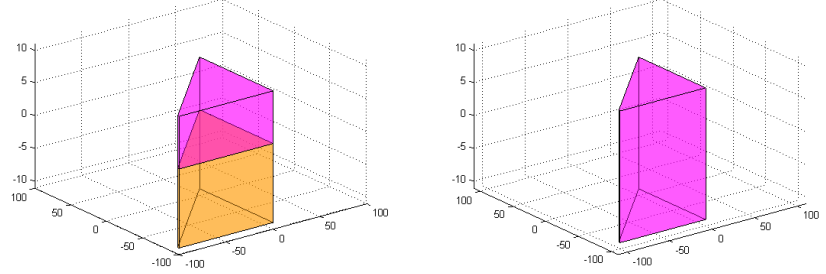

Figure 4. Merging of modes $\mathcal{A}_{7}, \mathcal{A}_{8}$ associated to the second dynamics

Starting from a total of 8 modes we have obtained a reduced system with just 3 modes. The results have been obtained by applying a greedy exploration, that, in this case, perform as well as the exhaustive exploration. The case of the greedy exploration performing worse than the exhaustive one is shown in Figure 5, where we associated mode $\mathcal{A}_{8}$ to the first dynamics and mode $\mathcal{A}_{6}$ to the second dynamics. In this case the reduction returns a total of 5 modes.
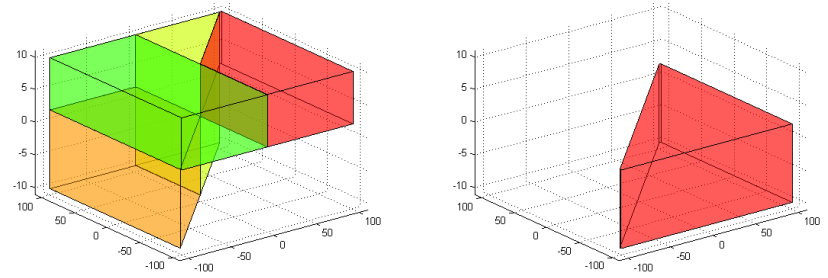

Figure 5. Greedy exploration: the modes associated to the first dynamics (on the left) are merged in a total of 4 regions

\section{CONCLUSIONS}

In this paper we introduced an approach to model reduction of discrete time hybrid systems that preserves the input/output 
behavior. The proposed approach rests on a sufficient condition for the unobservable part of the affine dynamics entering the MLD model description to remain unobservable when accounting for the hybrid system evolution. Our aim was reproducing the input/ouput behavior, irrespectively of the system initialization. If the system initialization were exactly known or confined to some region, the model reduction procedure could account for this additional information and, possibly, further reduce the model. This would be the case for linear systems. In the MLD systems framework, some combinations of the $\delta$ auxiliary variable that define the switching between modes in the PWA form might be pruned out because not admissible, which will possibly simplify the inequalities in the MLD representation. This requires further work.

\section{REFERENCES}

[1] A. Bemporad. Efficient conversion of mixed logical dynamical systems into an equivalent piecewise affine form. IEEE Transactions on Automatic Control, 49(5):832-838, 2004.

[2] A. Bemporad, G. Ferrari-Trecate, and M. Morari. Observability and Controllability of Piecewise Affine and Hybrid Systems. IEEE Transactions on Automatic Control, 45(10):1864-1876, 2000.

[3] A. Bemporad and N. Giorgetti. Logic-based solution methods for optimal control of hybrid systems. Automatic Control, IEEE Transactions on, 51(6):963-976, 2006.

[4] A. Bemporad and M. Morari. Control of systems integrating logic, dynamics, and constraints. Automatica, 35(3):407-427, 1999.

[5] A. Bemporad and M. Morari. Predictive Control of Constrained Hybrid Systems. In Nonlinear Model Predictive Control, pages 71-98, Ascona, Switzerland, 2000.

[6] Alberto Bemporad and Manfred Morari. Verification of hybrid systems via mathematical programming. In Hybrid Systems: Computation and Control, pages 31-45. Springer, 1999.

[7] Stephen P Boyd, Laurent El Ghaoui, Eric Feron, and Venkataramanan Balakrishnan. Linear matrix inequalities in system and control theory, volume 15. SIAM, 1994.

[8] G. Ferrari-Trecate and M. Gati. Computation observability regions for discrete-time hybrid systems. In Proceedings of the 42nd IEEE Conference on Decision and Control, volume 2, pages 1153-1158, Dec 2003.

[9] W.P.M.H. Heemels, B. De Schutter, and A. Bemporad. Equivalence of hybrid dynamical models. Automatica, 37(7):1085-1091, July 2001.

[10] M. Herceg, M. Kvasnica, C.N. Jones, and M. Morari. Multi-Parametric Toolbox 3.0. In Proc. of the European Control Conference, pages 502510, Zürich, Switzerland, July 17-19 2013. http://control.ee.ethz.ch/ $\sim$ mpt.

[11] Mihaly Petreczky. Realization theory of linear hybrid systems. In Hybrid Dynamical Systems, volume 457, pages 59-101. 2015.

[12] Mihály Petreczky and Jan H. van Schuppen. Observability reduction of piecewise-affine hybrid systems. In 19th Int. Symposium on Mathematical Theory of Networks and Systems, pages 203-210, 2010.

[13] Fabio Danilo Torrisi. Modeling and reach-set computation for analysis and optimal control of discrete hybrid automata. $\mathrm{PhD}$ thesis, Diss., Technische Wissenschaften ETH Zürich, Nr. 15064, 2003, 2003.

[14] Fabio Danilo Torrisi and Alberto Bemporad. Hysdel-a tool for generating computational hybrid models for analysis and synthesis problems. Control Systems Technology, IEEE Transactions on, 12(2):235-249, 2004.

[15] R. Vignali, L. Deori, and M. Prandini. Control input design: detecting non influential inputs while satisfying a reachability specification. In 19th World Congress of the International Federation of Automatic Control, Cape Town, South Africa, August 2014.

[16] R. Vignali and M. Prandini. Input design for a cascading system: An approach based on system decomposition and non-influential input detection. In 2014 IEEE Multi-Conference on Systems and Control, Antibes, France, October 2014 